دراسات في آثار الوطن العربي 16

\title{
Drei Göttinnen-Statuen aus Tell Basta im Ägyptischen Museum, Kairo
}

\author{
D. Mona M. Taha Hussein
}

\section{ABSTRACT}

The subject of this paper deals with three Goddess Statues made out of Egyptian faience, exhibited in P.19 E1 in the Egyptian Museum, Cairo, JE 43878-SR 3/7279, JE 43879-SR 3/7280, and JE 43880-SR 3/7281. They were all found in Bubastis, and were registered in the JE in 1913. Though similar in size, and style. Comparative studies with other statues showed that the statues JE 43878-SR 3/7279, JE 43879-SR 3/7280 could be dated to the 26 Dynasty.

\section{Einleitung:}

Die umfangreiche Sammlung der Göttinnen-Statuen im Ägyptischen Museum Kairo beherbergt drei stehende mischgestaltige Frauenstatuen (JE 43878, JE 43879, JE 43880) aus Tell Basta. Sie sind im Museumsinventar von 1913 registriert und im Raum P.19 E 1 im oberen Stockwerk ausgestellt ${ }^{1}$. Alle drei Statuen zeigen Bruchstellen, sind aber alle wieder zusammengesetzt und restauriert worden. Bei der Fig.1 JE 433878 ist ein Löwenkopf $\mathrm{zu}$ erkennen, der bei den beiden anderen Figuren (Fig.2 JE 433879 und Fig.3 JE 433880) fehlt. Die drei Statuen sind aus Ägyptischer Fayence ${ }^{2}$ und sind über 70

\footnotetext{
• Fakultät für Tourismus und Hotelmanagement, Oktober 6 Universität,Ägypten
}

${ }^{1}$ Ich möchte mich ganz herzlich bei Dr. Mahmoud el Halwagi, Direktor des Ägyptischen Museums Kairo und bei Herrn Sameh, Fotograf im Ägyptischem Museum Kairo, für ihre Unterstützung bedanken. Meinen Dank schulde ich auch Frau Dr. Dagmar Thesing, Leiterin der Kunsthalle Messmer in Riegel a.K. (Deutschland), für ihre Textkorrekturen und ihre verschiedenen Bemerkungen.

${ }^{2}$ In der JE werden (Quartz und Terre Emaille) als Materialen der drei Statuen genannt.“ Glazed pottery does not occur in Egypt prior to very late Roman or even early Arab Islamic times,'Dmount, ceramics, in: the Oxford Encyclopaedia of ancient Egypt, 2001, S.254. Quarzkeramik unterscheidet sich von Tonkeramik durch den Hauptbestandteil= 
دراسات في آثار الوطن العربي 16

$\mathrm{cm}$ hoch. Besonders interessant ist die Größe der Statuen. Seit Beginn des Alten Reiches ${ }^{3}$ bis in die Spätzeit hinein entstand eine Vielzahl von löwengestaltigen, vorwiegend weiblichen Gottheiten ${ }^{4}$. Göttinnen wie Sachmet, Bastet, Mehit, Menhit, Pachet, Tefnut und Schesemtet ${ }^{5}$ treten entweder in reiner Löwengestalt auf oder als mischgestaltige Feliden mit Frauenkörper und Löwenkopf, dessen Mähne im Grunde lediglich das männliche Tier kennzeichnet ${ }^{6}$ Allen Löwengöttinnen voran stehen Sachmet von Memphis und Bastet von Bubastis ${ }^{7}$. Seit dem Mittleren Reich ${ }^{8}$ werden die Eigenschaften der beiden Göttinnen stärker differenziert: Sachmet werden die kämpferischen, Bastet die friedfertigen Aspekte zugeschrieben. Der Darstellungstypus der Bastet als schreitende Göttin mit Katzenkopf lässt sich seit der Dritten Zwischenzeit belegen und hat sich während der Spätzeit und der griechisch-römischen Epoche ${ }^{9}$ zusehends etabliert. Die Angaben des Ägyptisches Museums über die drei mischgestaltigen Göttinnen-Statuen sind nicht ausreichend. Weder sind die Statuen datiert noch ist erwähnt, ob sie in einem Tempel oder im funeralem Kontext in Tell Basta/Bubastis gefunden wurden. Tell Basta liegt in der heutigen Großstadt Zagazig und wurde von den

$=$ Quarz (Sand), Wartke, Quarzkeramik in Vorderasien, in: Türkis und Azur. Quarzkeramik im Orient und Okzident, 1999, S.52.

${ }^{3}$ Zwei stehende mischgeschaltige Göttinnen-Statuen im Ägyptischen Museum Kairo, JE 99681 und JE 99682, Abusier, Altes Reich, 4. Dynastie, Zeit des Cheops, Terrakotta, über $90 \mathrm{~cm}$ hoch.

${ }^{4}$ Madeleine Page Gasser, Götter bewohnten Ägypten, Bronzefiguren der Sammlungen Bibel -Orient der Universität Freiburg Schweiz, 2001,S.34.

${ }^{5}$ Christian Leitz, Lexikon der ägyptischen Götter und Götterbezeichnungen, 2002, BII, S.739-S.743.

${ }^{6}$ Hönes, Sigrid Elke, Untersuchung zu den Wesen und Kult der Göttin Sachmet, 1976 S. 34),

${ }^{7}$ M. Saleh, H. Sourouzian, 1985, Katalog Die Hauptwerke im Ägyptischen Museum Kairo, Nr.254.

${ }^{8}$ D. Wildung, Staatliche Sammlung Ägyptischer Kunst, München Katalog, 1985, Nr.25, Stehende löwenköpfige Göttin, Amethyst, Höhe 6,2cm, Mittleres Reich, 12. Dynastie.

${ }^{9}$ Vaelska, Veit, Bubastis, Tell Basta in römischer Zeit, in: Archiv für Papyrusforschung und Verwandte Gebiete, 2009,S. 487 S- 498 Taf. XXXV. 
دراسات في آثار الوطن العربي 16

alten Ägyptern pr bas.t.t („Haus der Bastet“), von den Griechen „Bubastis“ genannt. ${ }^{10}$ Die Stadtanlage und die Tempel der Bastet wurden von Herodot, Manetho, Diodorus Siculus, Plinius Plutarch und Strabo erwähnt. Die Tempelanlage von Tell Basta wurde von Malus 1799 und später von Edouard Navielle (18871892) beschrieben ${ }^{11}$. Die Funde gingen seit Naville in verschiedene Museen ins Ausland und $\mathrm{zu}$ großen Teilen in das Ägyptische Museum Kairo. Die Bauten in Tell Basta lassen sich vom Alten Reich bis in die Spätzeit nachweisen. Die Stadt spielte eine bedeutende religiöse, wirtschaftliche und kulturelle Rolle. Die alte Stadt- und Tempelanlage wurde durch Kriege und Erdbeben zerstört. ${ }^{12}$ Der Handel mit Götterstatuen, die mit dem Tempelkult in Zusammenhang standen, wurde in der Spätzeit zunehmend wichtiger. ${ }^{13}$

\section{Material und Herstellung}

Alle drei Göttinnen-Statuen sind aus Ägyptischer Fayence hergestellt und damit aus einem Material, das sich durch einen weißen Scherben mit leuchtender, anfänglich blauer bis grüner Glasur des Soda-Kalk-Silica-Types auszeichnet. ${ }^{14}$ Das ägyptische Blau wurde im alten Ägypten Schesit genannt. ${ }^{15}$ Wie die anderen Werkstätten sind auch die Fayencebetriebe königliches Monopol und deshalb in Palast- oder Tempelnähe angesiedelt gewesen ${ }^{16}$. Das Wissen und die Techniken wurden vermutlich aus religiösen Gründen geheim gehalten. Die Rezepturen der

${ }^{10}$ Christian Tietze, Mahumud Omar Selim, Fünf Jahre archäologische Arbeit in der Tempelanlage von Tell Basta, in: Tell Basta Geschichte einer Grabung, 1997, S.27- S.29.

${ }^{11}$ Labib Habachi (1940-50), Shafik Farid (1961-67), Ahmed El-Sawi (1967-71), Mohamed Bakr (1980) und zuletzt die Arbeit in den Tempeln von Tell Basta, von den Zagazig und Potsdam Universität (1991-1996).

${ }^{12}$ Mohamed I. Bakr, Helmut Brandl, Egyptian Antiquities from KufurNigm and Bubastis, 2010 pp.160-161,pp.192-193.

13 Ibd. S. 190.

${ }^{14}$ Birgit Schlock Nolte, Ägyptische Fayence und Ägyptisch Blau im Alten Ägypten, in: Türkis und Azur, Quarzkeramik im Orient und Okzident, 1999, S.12- S.51, S.306.

${ }^{15} \mathrm{Ibd}, \mathrm{S} .51$.

${ }^{16}$ Erst 1938 wurden in der Lava des Vesuvs diese Kupferkalziumsilicate entdeckt, bei denen es sich um eine synthetische Form des natürlichen Minerals Cuprorivaithandelt, vgl. Nolte, S.19. 
دراسات في آثار الوطن العربي 16

Materialmischungen sind nicht erhalten. Es handelt sich bei der Fayenceherstellung um eine thermische Reaktion. Durch die moderne Forschung kennt man allein die Ausgangsstoffe und ihre Verarbeitungstechniken. Die verwendeten Rohstoffe der Fayence bestehen aus möglichst feinen Quarzsplitt, wie zerstoßenem Sand mit kaum 5\% natürlichen und beabsichtigen Beimengungen aus Tonerde, Kalk und Salzen wie Metalloxiden. Die Rohstoffe wurden mit Wasser verknetet, geformt, getrocknet und bei 800$1000{ }^{\circ} \mathrm{C}$ zu teilweise verglasenden Sinterkörpern gebrannt. Als Fließmittel wirken Natron und salzhaltige Pflanzenasche. Im Brand verursachen Mineralien, Kupferhammerschlag, Bronzeabfälle und Kupferoxide auf dem hellen Fayencekörper eine strahlend blaue Glasur. Fayence wird nur im kalten, ungebrannten Zustand geformt und anschließend im Ofen gebrannt.

\section{Beschreibung:}
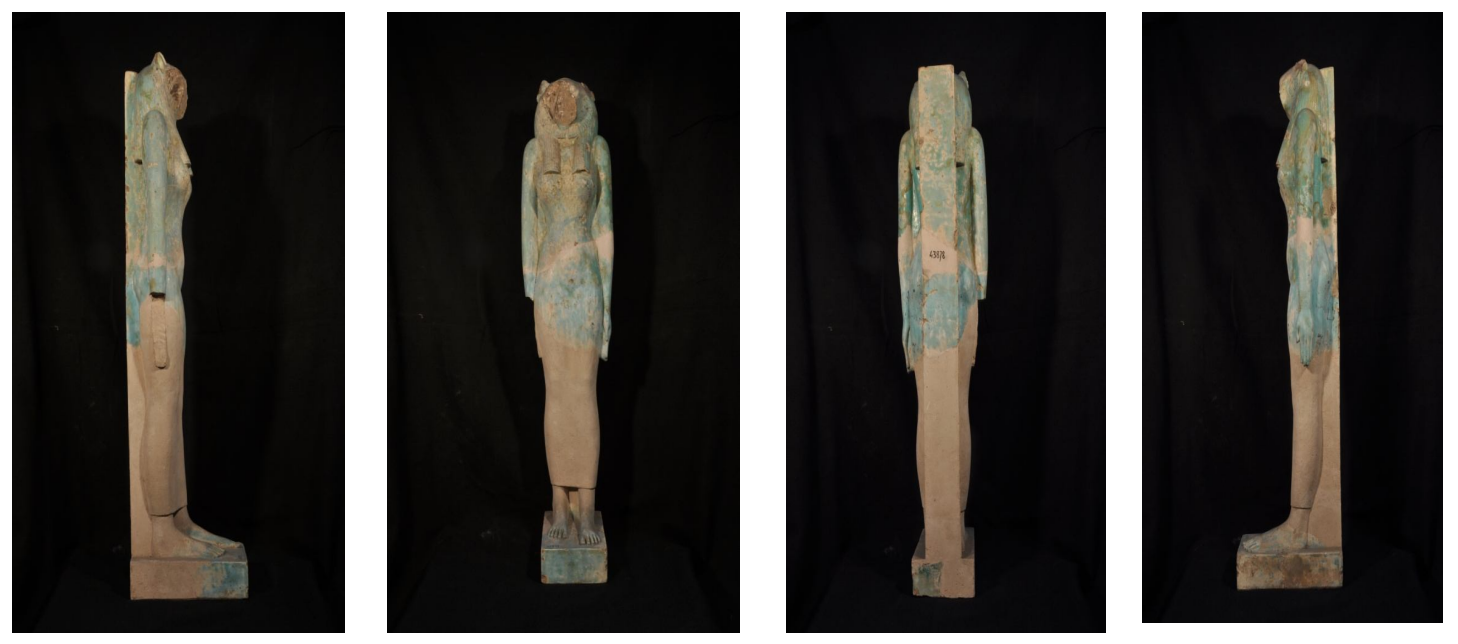

Fig.1. a,b,c,d: Mischgestaltige Frauenstaue mit Löwenkopf, stehend mit den linken Bein nach vorne dargestellt, JE, 43878SR 3/7279 (CSameh)

a. Die Statue stellt eine Frauengestalt mit Löwenkopf (Fig.1. a,b,c,d) JE, 43878- SR 3/7279 dar. Sie ist aus blauem Fayence 
دراسات في آثار الوطن العربي 16

hergestellt und $78 \mathrm{~cm}$ hoch. Die Bruchkante verläuft diagonal vom rechten Bein zum linken Oberschenkel, wobei die Statue wieder zusammengesetzt wurde. Das linke Ohr und die rechte Hand fehlen. Bestoßungen gibt es am Gesicht und an mehreren Stellen der Statue. Die blaue Oberfläche ist am unteren Teil der Statue kaum sichtbar. Sie steht mit dem linken Bein nach vorne auf einer rechteckigen Basis und ihre Gestalt ist in voller Höhe am Rückenpfeiler angelehnt. Die Statue ist unbeschriftet. Beide Arme sind lang nach unten gestreckt und liegen senkrecht an ihren Körper. Die Hände sind schlank und haben einen kurzen gewölbten Handrücken und lange Finger. Fingernägel und Nagelhaut sind angegeben. Die Zwischenräume zwischen Armen und Körper sowie Beinen und Rückenpfeiler sind ausgefüllt. Sie trägt ein enganliegendes faltenloses Gewand mit breiten Trägern, das bis zu den Knöcheln reicht. Ihr breiter Brustkorb hat volle Brüste, eine schmale Taille und runde Hüften. Der Nabel ist als tiefes Loch ausgebildet. Unterhalb der Taille wölbt sich ein kleiner Bauch. Die Scham ist in Form eines Dreiecks angedeutet. Das Antlitz des Löwen ist stets von einer dreiteiligen Strähnenperücke gerahmt, wobei den beiden vorderen Haarteilen eine bis $\mathrm{zu}$ den flauschigen Tierohren reichende Mähne vorgesetzt ist. Das Haupt ist unbekrönt. Die einzelnen parallelen Strähnen der Perücke sind als plastisch gestaltete Streifen ausgeformt. Der Brustkragen ist nur zwischen den beiden vorderen Perückenabschnitten wiedergegeben. Die Löwenohren ragen nur leicht über den Rand der Mähnen. Die Göttin trägt Arm- und Fußbänder. 

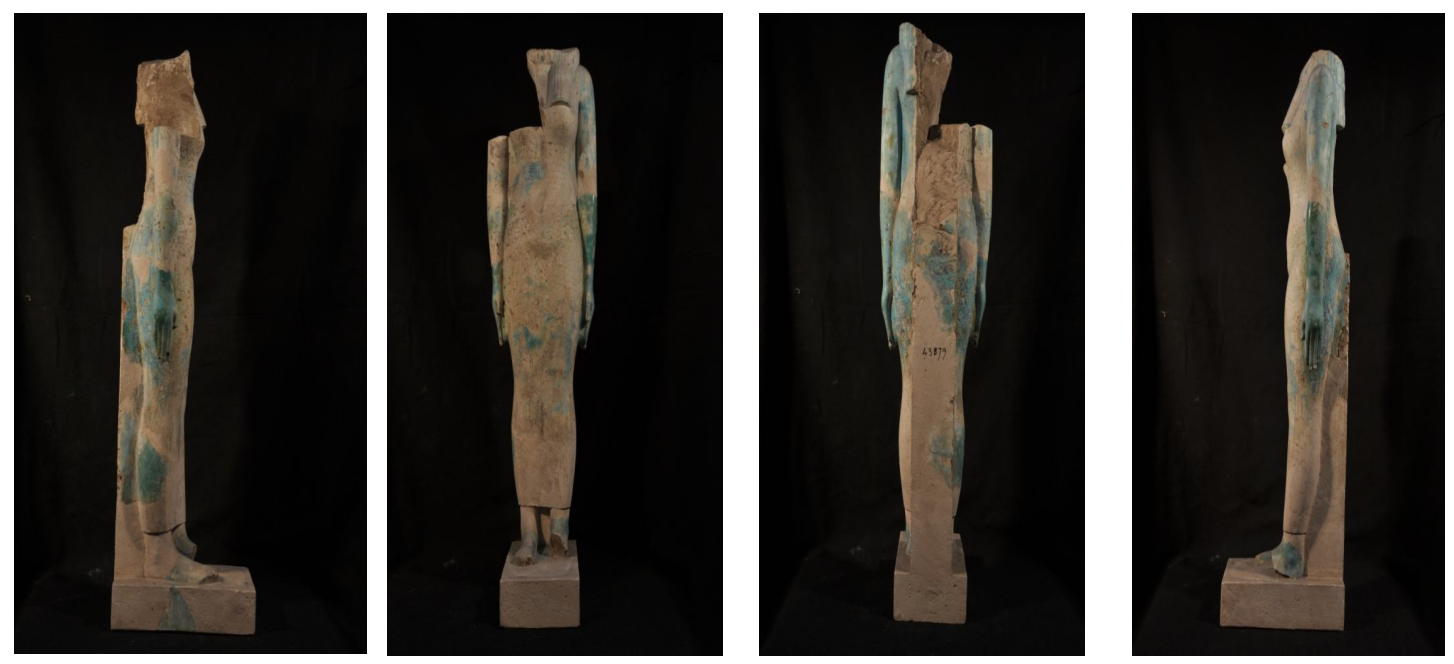

Fig.2, a, b, c, d: Stehende, mischgestaltige Frauenstatue mit Löwenkopf, JE 43879- SR 3/7280 (CSameh

b. Auch die zweite Statue stellt eine Frauengestalt mit Löwenkopf (Fig.2. a,b,c,d) JE, 43879- SR 3/7280 dar, ist aus blauem Fayence hergestellt und $75 \mathrm{~cm}$ hoch. Die Bruchkante, die diagonal vom rechten Bein zum linken Oberschenkel hochläuft, wurde ebenfalls wieder zusammengesetzt. Der Kopf, die rechte Schulter, die rechte Brust und der linke Fuß fehlen. Bestoßungen gibt es am Rückenpfeiler und an mehreren Stellen der Statue. Die blaue Fayenceoberfläche ist nur noch in Teilen erhalten, wovon besonders der untere Teil der Statue betroffen ist. Sie steht mit dem vorgesetzten linken Bein in der typischen Schrittstellung auf einer rechteckigen Basis, der Rückenpfeiler, an dem der Körper angelehnt ist, ist in der Mitte zerstört. Die Statue ist unbeschriftet. Die Arme sind lang und schlank und seitlich an ihren Körper gelegt. Die schlanken Hände haben einen kurzen gewölbten Handrücken und lange Finger mit ausgearbeiteten Fingernägeln und Nagelhäuten. Die Zwischenräume zwischen Armen und Körper sowie Beinen und Rückenpfeiler sind ausgefüllt. Sie trägt ein enganliegendes Federkleid mit breiten Trägern, das bis zu den Knöcheln reicht. Wie die Fig.1 hat ihr breiter Brustkorb volle Brüste, eine schmale Taille und runde 
دراسات في آثار الوطن العربي 16

Hüften. Auch der Nabel ist als tiefes Loch ausgebildet und unterhalb der Taille wölbt sich ein kleiner Bauch. Die Scham ist in Form eines Dreiecks angedeutet. Sie trägt die dreiteilige Strähnenperücke, deren beiden vorderen Partien auf der Brust ruhen. Die einzelnen parallelen Strähnen der Perücke sind als plastisch gestaltete Streifen ausgeformt. Der Brustkragen ist nur zwischen den beiden vorderen Perückenabschnitten wiedergegeben. Man kann noch einen Teil der Löwenmähne über dem Brustkragen erkennen. Die rechten Hand- und Fußgelenke sind mit Arm- und Fußbändern geschmückt.
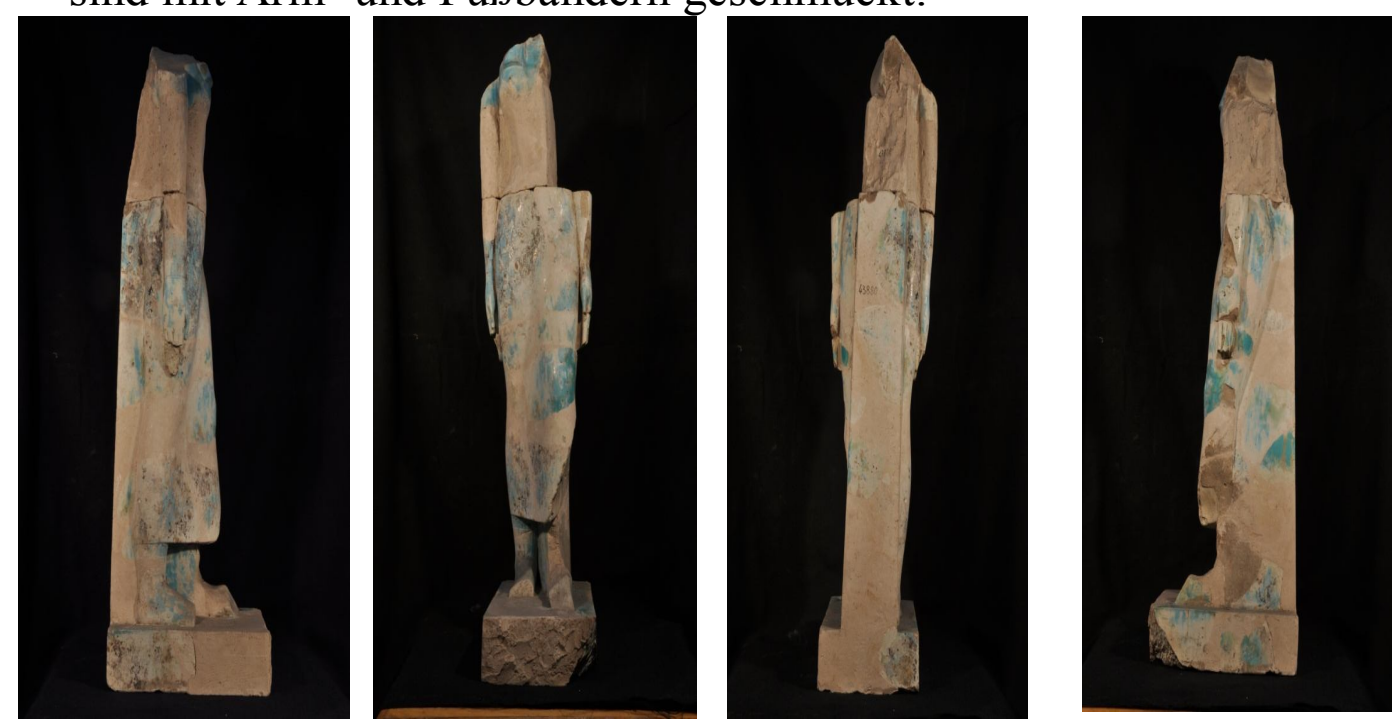

Fig.3, a, b, c, d: Schreitende Frauenstatue, JE 43880- SR 3/7281(CSameh)

c. Bei dieser Frauenstatue lässt der Erhaltungszustand keine genaue Zuschreibung zu. So ist es nicht gesichert, ob es sich bei der Gestalt um die Darstellung einer Göttin, Königin oder um eine Privatstatue handelt. Doch auch sie ist wie die beiden oben beschriebenen Statuen aus blauem Fayence hergestellt und $75 \mathrm{~cm}$ hoch. Kopf, Hals und die linke Hälfte des Oberkörpers fehlen. Sie trägt das enganliegende Kleid. Der kurze Oberkörper ist sehr schlank. Sie hat eine schmale, hochliegende Taille, gerundete Hüften und auffällig lange Oberschenkel. In der Profilansicht 
دراسات في آثار الوطن العربي 16

treten Bauch, Oberschenkel und besonderes das Gesäß starke hervor. Die Arme sind lang und schlank. Die schlanken Hände haben einen kurzen gewölbten Handrücken und lange Finger mit ausgearbeiteten Fingernägeln und Nagelhäuten. Die rechten Hand- und Fußgelenke sind mit Arm- und Fußbändern geschmückt, die abwechselnd mit senkrechten und waagrechten Streifen verziert sind. Die Statue lehnt, das linke Bein vorgestellt, an einem Rückenpfeiler, der bis zum unteren Ende des Hinterkopfes reicht.

\section{Kommentar}

In Tell Basta hat die ursprünglich dort ansässige Lokalgöttin Bastet aufgrund ihrer synkretistischen Verbindungen und der daraus resultierenden großen Beliebtheit sehr viel Verehrung gefunden. Auch Sachmet ist nicht nur in ihrer Hauptkultstätte Memphis verehrt worden, sondern auch in zahlreichen anderen Orten, u.a. auch in Tell Basta. Die drei Statuen sind aus Quartzkeramik (Ägyptischer Fayence), die sich von der europäischen Fayence unterscheidet, die aus Tonkeramik hergestellt wird. Wie im Stein lassen sich in der spätzeitlichen harten, feinen Fayence alle Details der Statuen naturalistisch modellieren $^{17}$. Zum Beispiel lassen sich am Kopf des Königs Psmatik (664v. Chr.) in Paris, Louvre N822, diese detaillierten Modellierungen in dem Hauch der blassen, hellgrünen Glasur sehr gut erkennen. Viele Skulpturen ${ }^{18}$ sind aus den besten, aber nicht mehr bekannten Werkstätten Unterägyptens erhalten. Die Anfänge für die enge Beziehung zwischen dem Löwen und dem Königtum findet man seit dem Alten Reich. Löwengestaltige Göttinnen wie Sachmet nehmen die Rolle von göttlichen Müttern wahr und sorgen sich um das Wohl des Königs. Aus der 4. Dynastie, der Zeit des Khufu, sind zwei über $90 \mathrm{~cm}$ große, aus

\footnotetext{
${ }^{17}$ Birgit Schlock Nolte, Ägyptische Fayence und Ägyptisch Blau im Alten Ägypten, in: Türkis und Azur, Quarzkeramik im Orient und Okzident, 1999, S. 46.

${ }^{18}$ Daressy G., Catalogue Général des AnitiqutésEgyptiennes du Musée du Caire, nos. CG 38001-39384, pl.L,Statues de divinités, 2 Bde., Le Caire 1905-1096.
} 
Terrakotta gefertigte löwenköpfige Göttinnen-Statuen erhalten geblieben, die sich heute im Ägyptischem Museum in Kairo (JE 99681 und JE 99682) befinden. Beide Statuen sind stehend dargestellt und lehnen sich an eine rechteckige Rückenplatte an. An der linken Seite der Rückenplatte der Statue JE 9982 ist der Name von König Cheops eingraviert. Sein Name attributiert die kleine Statue, die neben dem rechten Bein der löwenköpfigen Göttin steht und die ihren Finger im Mund steckt. Neben dem linken Bein der Göttin steht eine weitere kleine Staute mit dem Namen Pepi I. Sie ist vermutlich später, während der Regierungszeit dieses Königs, der Figurengruppe zugefügt worden und gibt so die Schutzfunktion der löwenköpfigen Göttinen zu erkennen. Da alle drei hier zur Diskussion stehenden Statuen (Fig1, 2 und 3) nicht beschriftet sind und keine besonderen Attribute tragen, durch sie sich von anderen Göttinnen unterscheiden ließen, ist eine gesicherte Identifizierung nicht möglich. Allerdings zeigt der Darstellungstypus aller drei Figuren große Übereinstimmung. Nur Fig. 2 weicht durch die Art ihrer Bekleidung stärker von den anderen beiden $a b$, denn sie trägt ein Federkleid. ${ }^{19}$ Doch am meisten unterscheidet sich Fig.3 hinsichtlich ihres Körperbaus von den anderen zwei Frauenstatuen. Auch wird aufgrund ihrer starken Beschädigung und des Fehlens ihres Kopfes eine gesicherte Zuschreibung verhindert und muss hier offen bleiben.

Insgesamt sprechen die Merkmale, die alle drei Statuen aufweisen und diese miteinander verbinden, für eine Datierung in die 26. Dynastie. So ist der Statuentypus Standfigur einer Frau im enganliegendem Trägerkleid und die Modellierung des Körpers mit langen Armen und Beinen, breitem Brustkorb, vollen Brüsten, der schmalen Taille und den langgezogenen, leicht

\footnotetext{
${ }^{19}$ Eine Statuette des Gottes Ptah des Ägyptischen Museums in Kairo, JE 60739, 62703 (Temp.29.3.3485), 18. Dynastie, aus Tutanchamuns Grab stellt den Gott stehend mit einem Federkleid dar. Eine aus den selben Grab stammende, thronende Sachmet-Statue im Ägyptischen Museum Kairo, JE 60749,62704 (temp.No.29.3.34.92) zeigt die Göttin mit einen Netzkleid bekleidet.
} 
دراسات في آثار الوطن العربي 16

gerundeten Hüften in der 26. Dynastie präsent. Dagegen gibt es keine Parallelen für das Trägerkleid in der ptolemäischen Plastik. $^{20}$ Besonders der beschriftete Torso der Gottesgemahlin Nitokris im Ägyptischem Museum in Kairo (JE 25582) sowie die Statuen der Göttin Mut im Charles Edwin Willbour Fund in Brooklyn (Inv.Nr. 76.38.) und der Göttin Isis in The Walter Art Gallery in Baltimore (Inv. Nr. 4.2121) zeigen die oben beschriebenen typischen Formen.

${ }^{20}$ Albermeiers Sabine, Untersuchungen zu den ptolemäischen Frauenstatuen, 2002, S. 274. Eva Rogge, Statuen 30. Dynastie und der Ptolemäischen Epoche, 11, 113-117. 


\section{ملخص البحث:}

بتناول البحث ثلاث تماثيل ألهات معروضة في قاعة P.19 E1 بالمتحف المصري

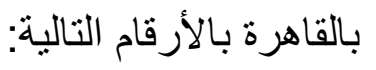

JE 43878-SR 3/7279, JE 43879-SR 3/7280, and JE 43880-SR 3/7281

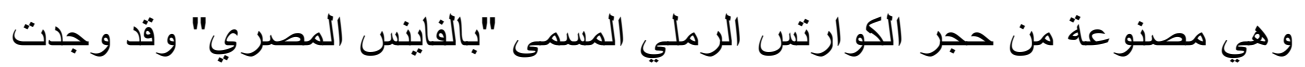

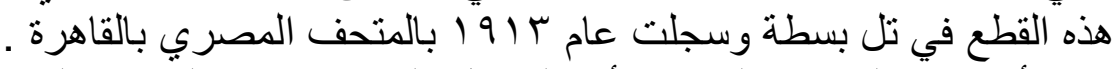

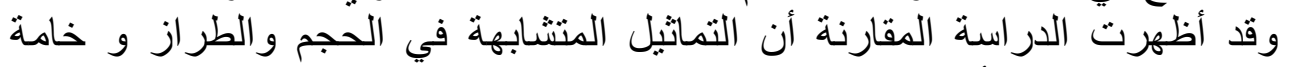

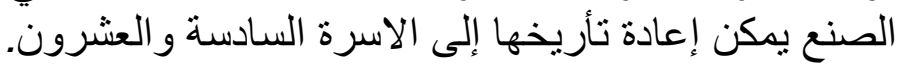

